Swarthmore College

Works

3-1-2010

\title{
Stable Spheromak Formation By Merging In An Oblate Flux Conserver
}

Timothy G. Gray , '01

Michael R. Brown

Swarthmore College, doc@swarthmore.edu

C. D. Cothran

G. Marklin

M. J. Schaffer

Follow this and additional works at: https://works.swarthmore.edu/fac-physics

Part of the Physics Commons

Let us know how access to these works benefits you

\section{Recommended Citation}

Timothy G. Gray , '01; Michael R. Brown; C. D. Cothran; G. Marklin; and M. J. Schaffer. (2010). "Stable Spheromak Formation By Merging In An Oblate Flux Conserver". Physics Of Plasmas. Volume 17, Issue 3. DOI: $10.1063 / 1.3334324$

https://works.swarthmore.edu/fac-physics/103

This work is brought to you for free by Swarthmore College Libraries' Works. It has been accepted for inclusion in Physics \& Astronomy Faculty Works by an authorized administrator of Works. For more information, please contact myworks@swarthmore.edu. 


\section{AIP | Physics of Plasmas}

\section{Stable spheromak formation by merging in an oblate flux conserver}

T. Gray, M. R. Brown, C. D. Cothran, G. Marklin, and M. J. Schaffer

Citation: Physics of Plasmas (1994-present) 17, 032510 (2010); doi: 10.1063/1.3334324

View online: http://dx.doi.org/10.1063/1.3334324

View Table of Contents: http://scitation.aip.org/content/aip/journal/pop/17/3?ver=pdfcov

Published by the AIP Publishing

\section{Articles you may be interested in}

Validation of single-fluid and two-fluid magnetohydrodynamic models of the helicity injected torus spheromak experiment with the NIMROD code

Phys. Plasmas 20, 082512 (2013); 10.1063/1.4817951

Relaxation of spheromak configurations with open flux

Phys. Plasmas 16, 112508 (2009); 10.1063/1.3264077

Dipole trapped spheromak in a prolate flux conserver

Phys. Plasmas 13, 102503 (2006); 10.1063/1.2356690

Stable high beta spheromak equilibria using concave flux conservers

Phys. Plasmas 7, 2959 (2000); 10.1063/1.874147

Scaling studies of spheromak formation and equilibrium

Phys. Plasmas 5, 1027 (1998); 10.1063/1.872632

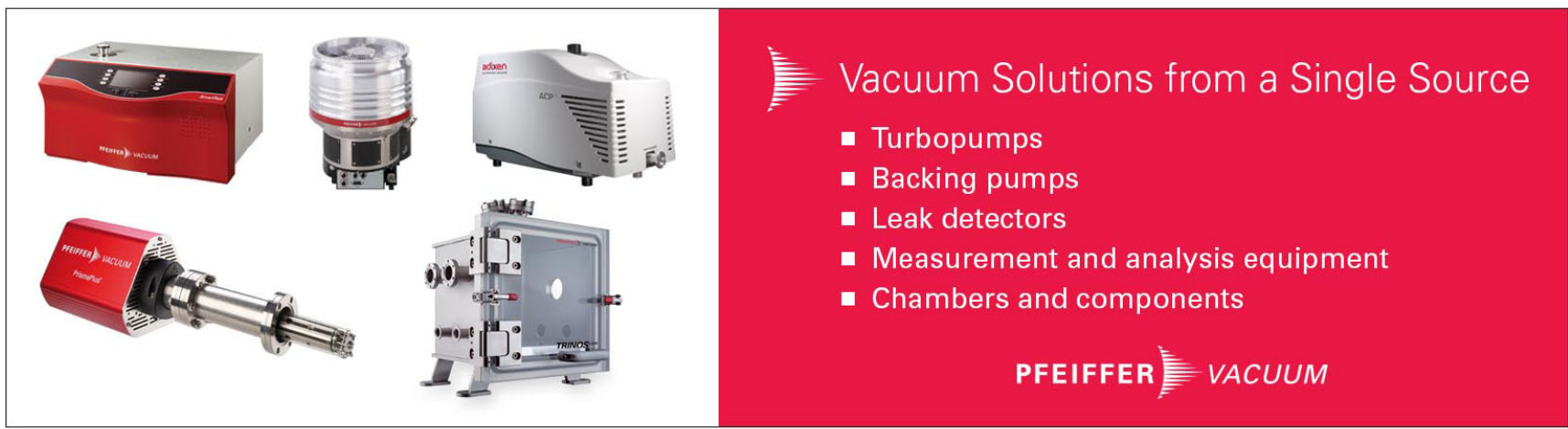




\title{
Stable spheromak formation by merging in an oblate flux conserver
}

\author{
T. Gray, ${ }^{1, a)}$ M. R. Brown, ${ }^{1}$ C. D. Cothran, ${ }^{1, b)}$ G. Marklin, ${ }^{2}$ and M. J. Schaffer ${ }^{3}$ \\ ${ }^{1}$ Department of Physics and Astronomy, Swarthmore College, Swarthmore, Pennsylvania 19081-1397, USA \\ ${ }^{2}$ PSI-Center, University of Washington, Seattle, Washington 98195-2250, USA \\ ${ }^{3}$ General Atomics, P.O. Box 85608, San Diego, California 92186-5608, USA
}

(Received 18 August 2009; accepted 4 February 2010; published online 29 March 2010)

\begin{abstract}
An axisymmetric spheromak formed by the dynamic merging of two smaller spheromaks of the same magnetic helicity in the Swarthmore Spheromak Experiment (SSX) [M. R. Brown, Phys. Plasmas 6, 1717 (1999)] has been observed and characterized. The spheromak is formed in an oblate (tilt stable), trapezoidal, $6 \mathrm{~mm}$ wall copper flux conserver in SSX, which is $0.5 \mathrm{~m}$ in diameter and $L=0.4 \mathrm{~m}$ in length at its largest dimensions. This configuration is formed by cohelicity merging of two spheromaks (either both right-handed or both left-handed) in which the merging poloidal fluxes are parallel (i.e., no field reversal for reconnection to occur initially). After a period of dynamic and nonaxisymmetric activity, the configuration ultimately relaxes to an axisymmetric state. A nonaxisymmetric tilted state, very close in total energy to the axisymmetric state, is also sometimes observed. This configuration is characterized by a suite of magnetic probe arrays for magnetic structure $\boldsymbol{B}(\boldsymbol{r}, t)$, ion Doppler spectroscopy for $T_{i}$ and flow, and interferometry for $n_{e}$. The magnetic structures of both states match well to computed eigenstates. (c) 2010 American Institute of Physics. [doi:10.1063/1.3334324]
\end{abstract}

\section{INTRODUCTION}

Spheromaks ${ }^{1}$ are compact, translatable plasma configurations with a simply connected boundary typically formed in a close-fitting flux conserver with a single source. ${ }^{2-5}$ Merging of spheromaks was developed by $\mathrm{Ono}^{6}$ and implemented at the Swarthmore Spheromak Experiment $(\mathrm{SSX})^{7,8}$ but typically spheromaks of opposite helicity (one righthanded and the other left handed) are merged in order to form a field-reversed configuration (FRC). This counterhelicity merging resulted in plasma heating due to reconnection in the merging layer and a route to high plasma $\beta$.

An axisymmetric spheromak formed by the dynamic merging of two smaller spheromaks of the same magnetic helicity in SSX has been observed and characterized. The merging is performed in an oblate (tilt stable), trapezoidal, 6 $\mathrm{mm}$ wall copper flux conserver in SSX, which is $0.5 \mathrm{~m}$ in diameter and $L=0.4 \mathrm{~m}$ in length at its largest dimensions. Cohelicity merging in SSX does not involve an initial state where reconnection of poloidal fields can occur while still maintaining axisymmetry (at least not initially). The toroidal fields of the spheromaks are oppositely directed, while the poloidal fields are codirected and form a radial cusplike structure at the midplane. Despite the absence of a field reversal at which reconnection can commence, the system relaxes to the minimum energy state subject to the constraint of maintaining two units of helicity, passing through an intermediate nonaxisymmetric phase which is necessary for reconnection to occur. This state in our oblate flux conserver is an axisymmetric spheromak. This is a clear example of Taylor relaxation in a magnetohydrodynamics (MHD) system. ${ }^{9}$

The SSX device is outfitted with an oblate flux con-

\footnotetext{
${ }^{a)}$ Electronic mail: tgray1@swarthmore.edu.

${ }^{b)}$ Present address: Global Strategies Group, Crofton, MD 21114.
}

server. The flux conserver is $0.5 \mathrm{~m}$ in diameter and $L$ $=0.4 \mathrm{~m}$ in length, and is constructed out of $6 \mathrm{~mm}$ thick copper. The flux conserver has a longer entrance region compared with previous SSX designs. The merging region is not right cylindrical but is a trapezoid of revolution in shape. The flux conserver is depicted in the schematic of the SSX device shown in Fig. 1, along with a calculated spheromak equilibrium. This shape, which has flared entrance regions between the ends of the coaxial plasma guns and the main flux conserver, was chosen to allow a wide opening for two spheromaks to enter the flux conserver.

Global magnetic structure of objects formed in SSX is studied with up to 600 individual internal magnetic probes. ${ }^{10}$ Both compact, high spatial resolution arrays, as well as distributed, coarse resolution arrays have been used. For the work presented here, linear arrays of quartz jacketed probes

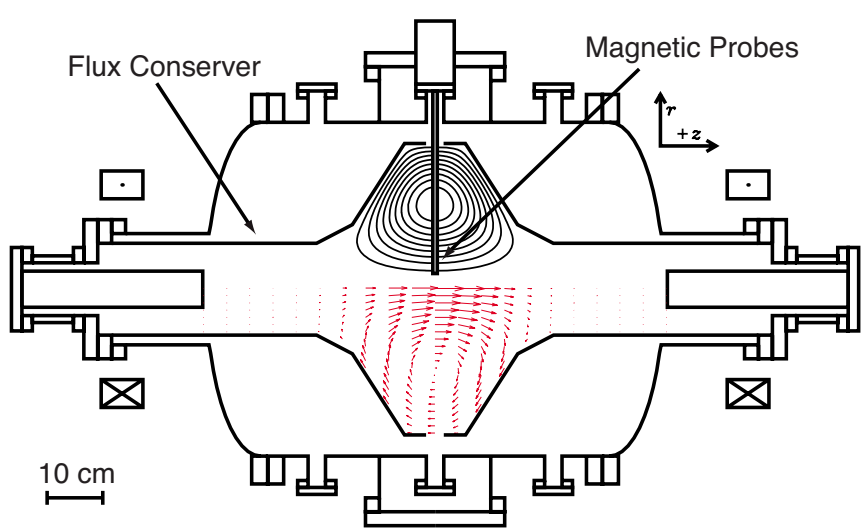

FIG. 1. (Color online) The conical oblate flux conserver and a calculated spheromak equilibrium are shown in the SSX vacuum vessel. The poloidal field of the equilibrium is represented by vectors, while the contours represent flux surfaces. The quartz-clad magnetic probes are also indicated. 


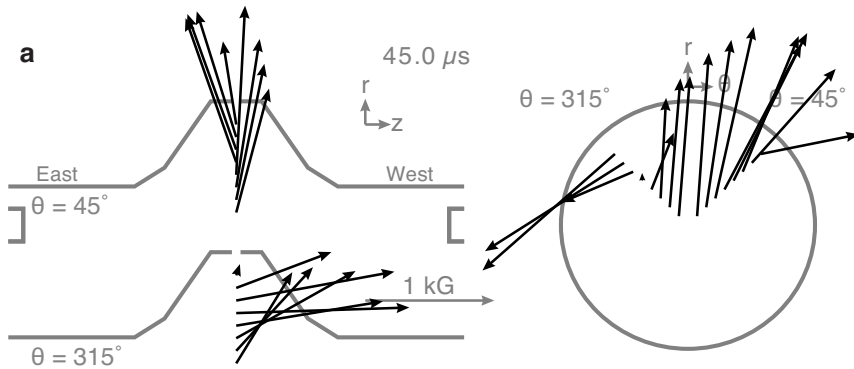

b

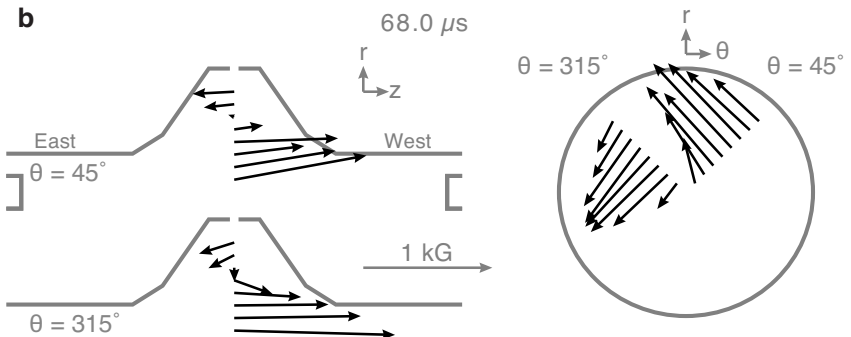

FIG. 2. Magnetic data. The probe displayed in the top left of each figure is installed at $\theta=45^{\circ}$, while the probe shown in lower left is at $\theta=315^{\circ}$. (a) is early in the discharge during the merging phase at $45 \mu \mathrm{s}$. It is clear that this phase is dynamic and nonaxisymmetric. Later in the discharge at $68 \mu \mathrm{s}$, shown in (b), the merging has resulted in an axisymmetric spheromak.

are used. Line averaged electron density for each configuration is monitored with quadrature $\mathrm{HeNe}$ laser interferometer. ${ }^{8}$ For these studies, the single interferometer chord is placed on a diameter at the midplane.

In addition, line averaged ion flow and heating $T_{i}$ at the midplane is monitored with a $1.33 \mathrm{~m}$ ion Doppler spectrometer (IDS). ${ }^{11}$ High spectral resolution $(\Delta v=5 \mathrm{~km} / \mathrm{s})$ is achieved with an Echelle grating operating at 25th order. High temporal resolution is achieved by using a 32 channel photomultiplier tube array. The SSX IDS instrument measures with $1 \mu$ s or better time resolution the width and Doppler shift of the $\mathrm{C}_{\mathrm{III}}$ impurity ( $\mathrm{H}$ plasma) $229.7 \mathrm{~nm}$ line to determine the temperature and line-averaged flow velocity during spheromak merging events.

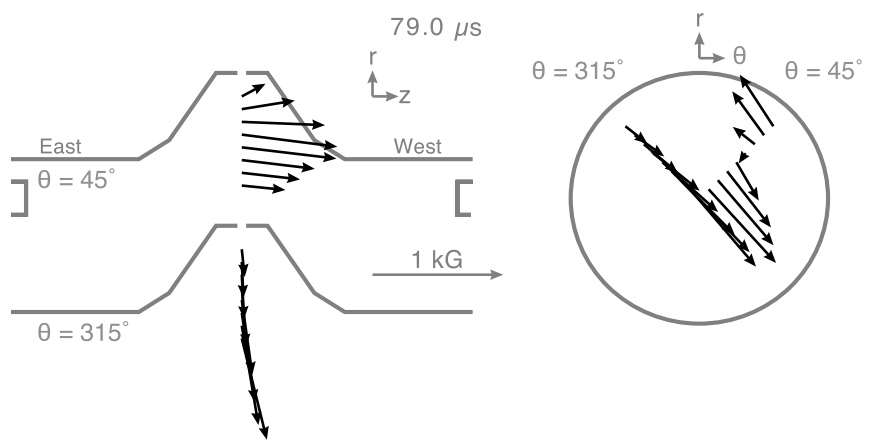

FIG. 3. Magnetic data from the tilted mode. Orientation is the same as in Fig. 2. Much like fields from the axisymmetric state, the fields are very dynamic early in time. Later in time, they settle down into the tilted configuration shown, with the geometric axis of the spheromak aligned with the probe located at $315^{\circ}$.
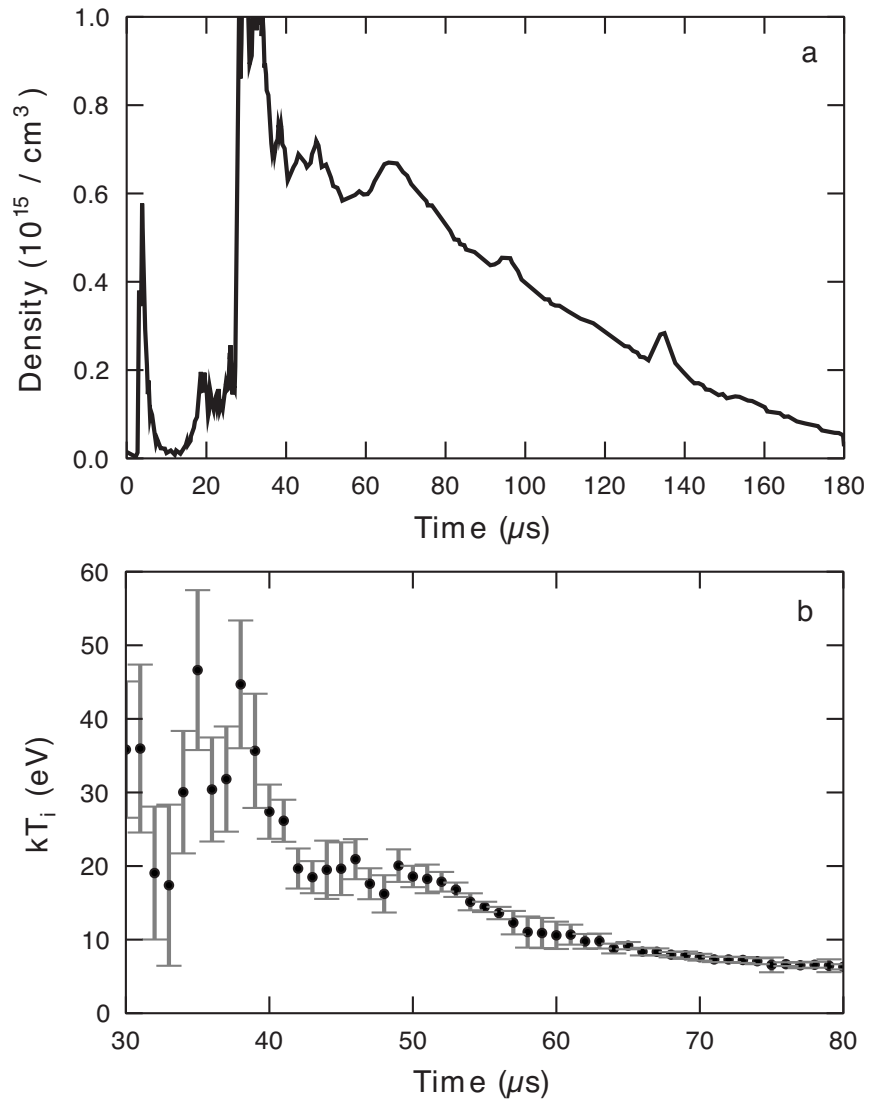

FIG. 4. Typical plasma temperatures and densities for the cohelicity merged object. Note the time scales are not the same. In (a), a typical line integrated density $\left(\bar{n}_{e}\right)$ is shown. In the main region of the discharge $(40-120 \mu \mathrm{s})$, densities of $1-6 \times 10^{14} / \mathrm{cm}^{3}$ are seen. (b) shows the ion temperature as measured by the IDS system, averaged over 16 shots. While there is an initial spike in $T_{i}$ during the merging phase of the discharge $(t$ $=30-50 \mu \mathrm{s}), T_{i}$ decays slowly. The error bars represent the spread in the temperatures of the 16 shots.

\section{RESULTS}

The spheromak state resulting from the cohelicity merging of the two initial spheromaks is a long-lived state, longer than a single gun-produced spheromak. The magnetic structure measured with two linear magnetic probe arrays (early

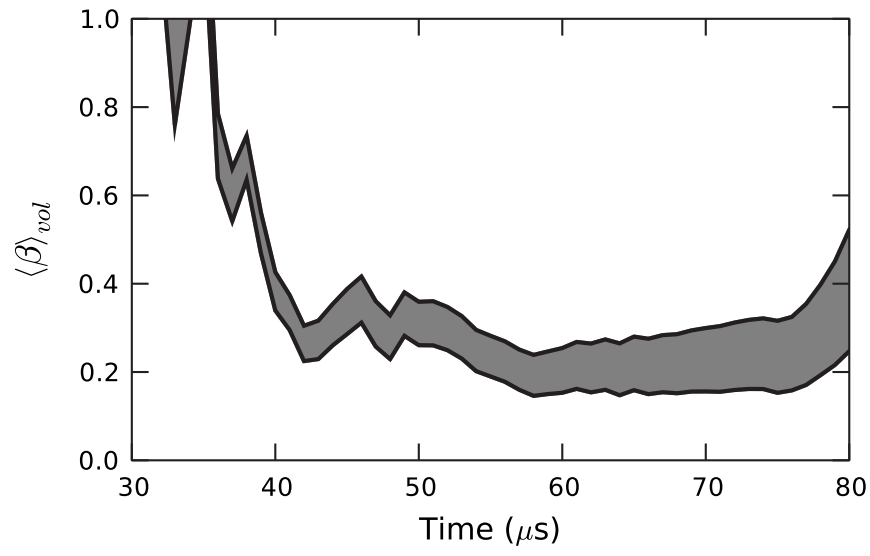

FIG. 5. Volume averaged beta $\left(\langle\beta\rangle_{\text {vol }}\right)$ for the merged object. The range of $\langle\beta\rangle_{\mathrm{vol}}$ displayed corresponds $T_{e}=0-7 \mathrm{eV}$. After merging is completed around $50 \mu \mathrm{s},\langle\beta\rangle_{\mathrm{vol}}$ settles to a value of $0.2-0.3$. 

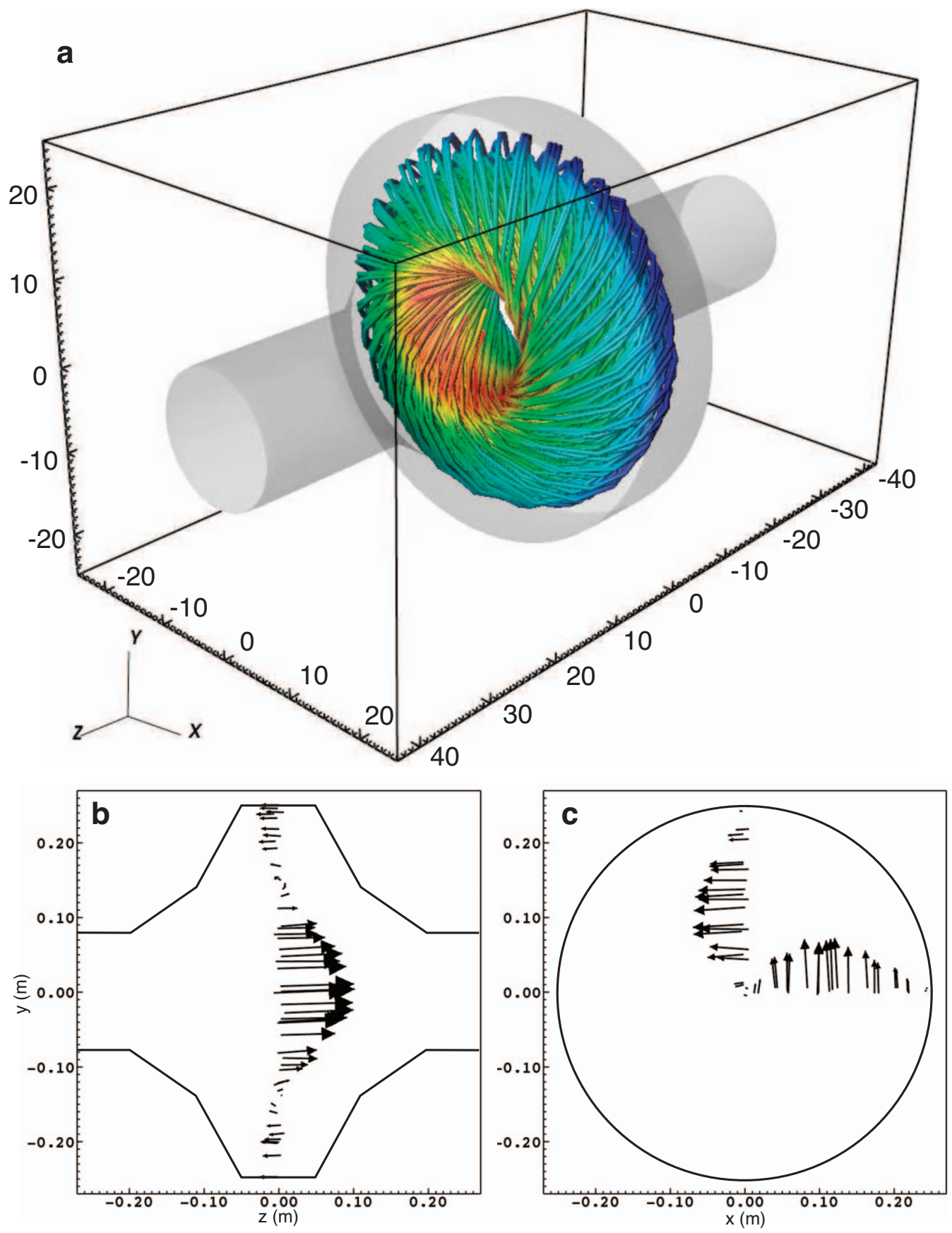

FIG. 6. (Color) The lowest eigenstate for Taylor relaxation in the oblate flux conserver in SSX, generated by the PSI-TET code. In (a), the flux conserver geometry is represented by the gray transparent solid, while the streamlines illustrate the magnetic fields, showing the overall structure of the state. Color represents $|\boldsymbol{B}|$. Magnetic field vectors are displayed in [(b) and (c)] with the poloidal field shown in (b) and the toroidal field in (c) compared with the measured field in Fig. 2(b).

and late in time) is depicted in Fig. 2. Early in time during the merging phase of the discharge $(t=30-50 \mu \mathrm{s})$, as shown in Fig. 2(a), the magnetic field vectors are very dynamic and nonaxisymmetric. After the merging has completed, shown in Fig. 2(b), the resulting plasma is a long-lived, axisymmetric spheromak, with a lifetime of $>120 \mu$ s. This state is longer lived compared with the typical plasmas in SSX, which last $80-100 \mu \mathrm{s}$. This stable axisymmetric state can be obtained by merging two left-handed spheromaks or two right-handed spheromaks. The final state can be aligned such that its poloidal flux on axis pointing either east or west.
Since the final spheromak does not consistently prefer either of the orientations, systematic differences between the two merging spheromaks can be ruled out as influencing the final equilibrium. It should be noted that some of the final merged spheromaks tilt early in time, as shown in Fig. 3 and discussed in the next section.

A range of densities is attainable in this configuration. Figure 4(a) shows the typical evolution of $n_{e}(t)$. Line integrated densities, as measured by an $\mathrm{HeNe}$ interferometer with a range of $1-6 \times 10^{14} / \mathrm{cm}^{3}$. This is in the range of densities of single spheromaks and FRCs previously created in 


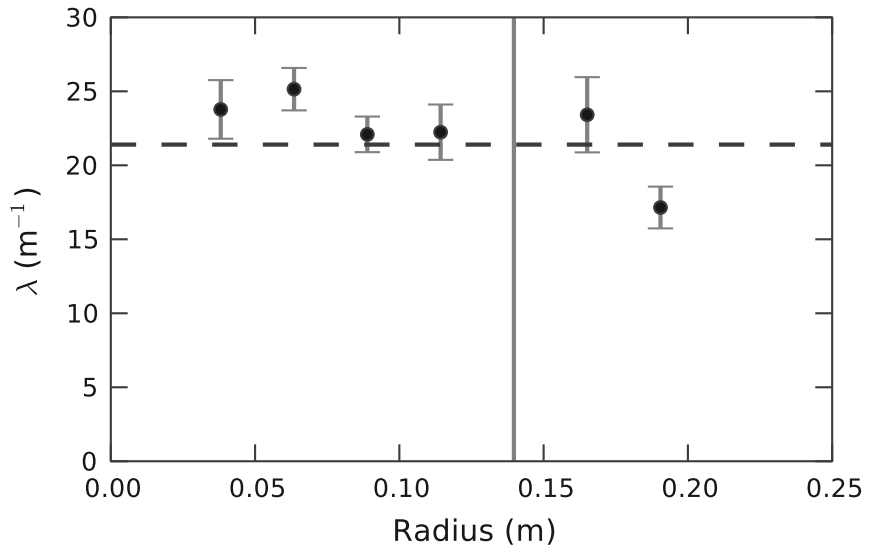

FIG. 7. $\lambda$ profile for the axisymmetric state. The profile is flat. The calculated value from PSI-TET is marked by the dashed line with a value of $\lambda$ $=21.4 \mathrm{~m}^{-1}$. The large error bar for the point at $r=0.14 \mathrm{~m}$ is due to the very small value of $B_{z}$ at that location.

the SSX device. All density traces show a slow decay in density starting at 55-65 $\mu$ s and lasting for more than $100 \mu \mathrm{s}$.

Ion temperatures measured by the IDS diagnostic reveal a relatively low temperature spheromak as result of the cohelicity merging. The turbulent initial merging process has a moderate $T_{i} \approx 35 \mathrm{eV}$, presumably heating from reconnection, and rapidly cools to $T_{i} \approx 10 \mathrm{eV}$, as seen in Fig. 4(b). The temperatures shown in Fig. 4(b) are calculated by a weighted average over 16 shots. The weighting used is the error of the calculated temperature for a given shot. The error bars represent the spread in the temperatures of the 16 shots.

Volume averaged beta $\left(\langle\beta\rangle_{\mathrm{vol}}\right)$ for the merged object is shown in Fig. 5. After merging ends at $50 \mu \mathrm{s},\langle\beta\rangle_{\mathrm{vol}}$ stabilizes at 0.3 . The $\langle\beta\rangle_{\text {vol }}$ measurement is calculated by using $T_{i}$ from the IDS measurements, $T_{e}$ in the range of $0-7 \mathrm{eV}$, and the average $B$ from the magnetic probes. The technique for measuring $T_{e}$ on SSX uses the ratio of the $\mathrm{C}_{\mathrm{III}}$ line at 97.7 $\mathrm{nm}$ and the $\mathrm{C}_{\mathrm{IV}}$ line at $155 \mathrm{~nm}$, measured with a vacuum ultraviolet spectrometer. ${ }^{12}$ This method is insensitive to $T_{e}$ values below $\sim 7 \mathrm{eV} . T_{e}$ for the merged object was either at or below this threshold, $T_{e} \leq 7 \mathrm{eV}$.

Counterhelicity merging experiments have also been performed in order to form null helicity FRCs in this oblate geometry but it is found that the approach to magnetic equilibrium is much more robust in the cohelicity experiments. The present results suggest that in the MHD regime, formation of FRCs by counterhelicity merging may be a sensitive process. Modeling is currently underway to shed light on the counterhelicity merging process in this geometry.

\section{DISCUSSION}

Minimum energy eigenstates for $\nabla \times \boldsymbol{B}=\lambda \boldsymbol{B}$ were originally calculated in Ref. 13 . The relaxation process by which these states might be reached via magnetic reconnection, while conserving magnetic helicity was proposed in Ref. 9 in an effort to explain the self-reversal in the reversed field pinch (RFP). Based on the past successes of Taylor relaxation theory at explaining the RFP puzzle and the more con-

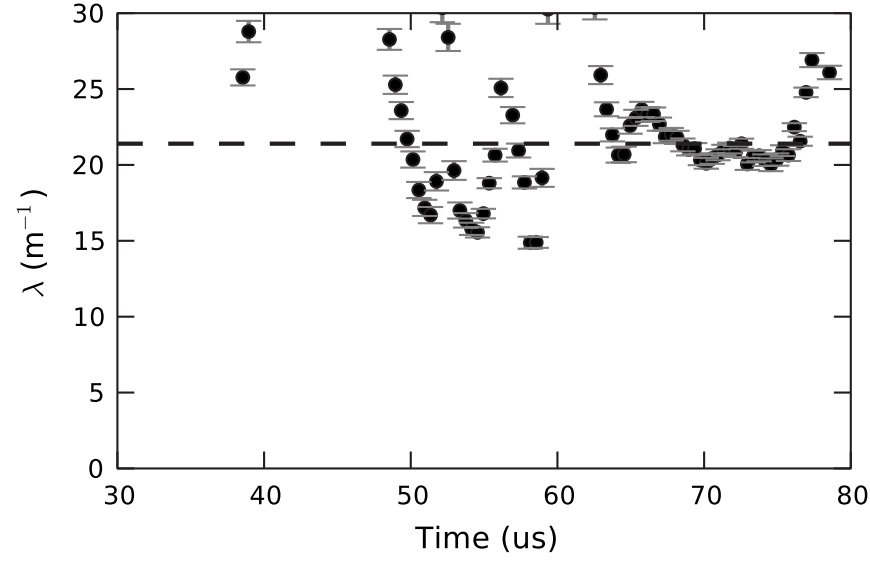

FIG. 8. The time evolution of $\lambda$ for the axisymmetric state. The calculated value for the geometry is marked by the dashed line with a value of $\lambda$ $=21.4 \mathrm{~m}^{-1}$. The plasma is in the relaxed state during the period at 64-78 $\mu \mathrm{s}$.

ventional kinds of spheromak formation, one would expect Taylor relaxation to function as well for cohelicity merging of two spheromaks aligned conventionally in which a magnetic field reversal exists between them. This has been demonstrated in Ref. 14. However, the SSX cohelicity initial state has no field null where reconnection can initially commence, while maintaining axisymmetry and no obvious initial path for reconnection, and certainly no guarantee that any relaxation that might occur should approximately conserve helicity. Despite this, spheromak formation by cohelicity merging in SSX is a robust process, always resulting in a spheromak configuration.

Our cohelicity produced spheromaks are approximately axisymmetric, but they clearly have some nonaxisymmetry present. This has been observed in prior spheromak experiments in otherwise tilt-stable geometries. ${ }^{15}$ Unlike previous counterhelicity merging in SSX in a prolate flux conserver, ${ }^{7}$ the merging phase of the two cohelicity spheromaks is highly dynamic, random, and nonaxisymmetric. It is important to note that in order to reach the axisymmetric final state, an intermediate nonaxisymmetric phase must occur. Excitation of high order spatial and temporal modes seems to be critical during the merging process of the two spheromaks, as the initial cusplike structure formed by the merging spheromaks must ultimately reconnect and relax in order to form a stable axisymmetric final state.

Using the PSI-TET code, ${ }^{16}$ eigenmodes for the Taylor relaxation of a plasma with a finite helicity in a arbitrary three-dimensional geometry can be calculated. Output from PSI-TET for the lowest eigenmode, with $\lambda=21.4 \mathrm{~m}^{-1}$, for the oblate geometry in SSX is shown in Fig. 6. This axisymmetric mode is very similar to the final merged spheromak formed in SSX. The magnetic vectors sampled at the same locations as the magnetic probes in SSX, shown in Fig. 2, show almost identical profiles. $\lambda$ is calculated from the experimentally measured magnetic field using

$$
\lambda=\frac{1}{B_{z}}\left[\frac{1}{r} \frac{\partial}{\partial r}\left(r B_{\theta}\right)+\frac{1}{r} \frac{\partial B_{r}}{\partial \theta}\right] .
$$

It should be noted that at earlier times, axisymmetry (or a pure $m=1$ mode) cannot be assumed so the values of $\lambda$ can- 

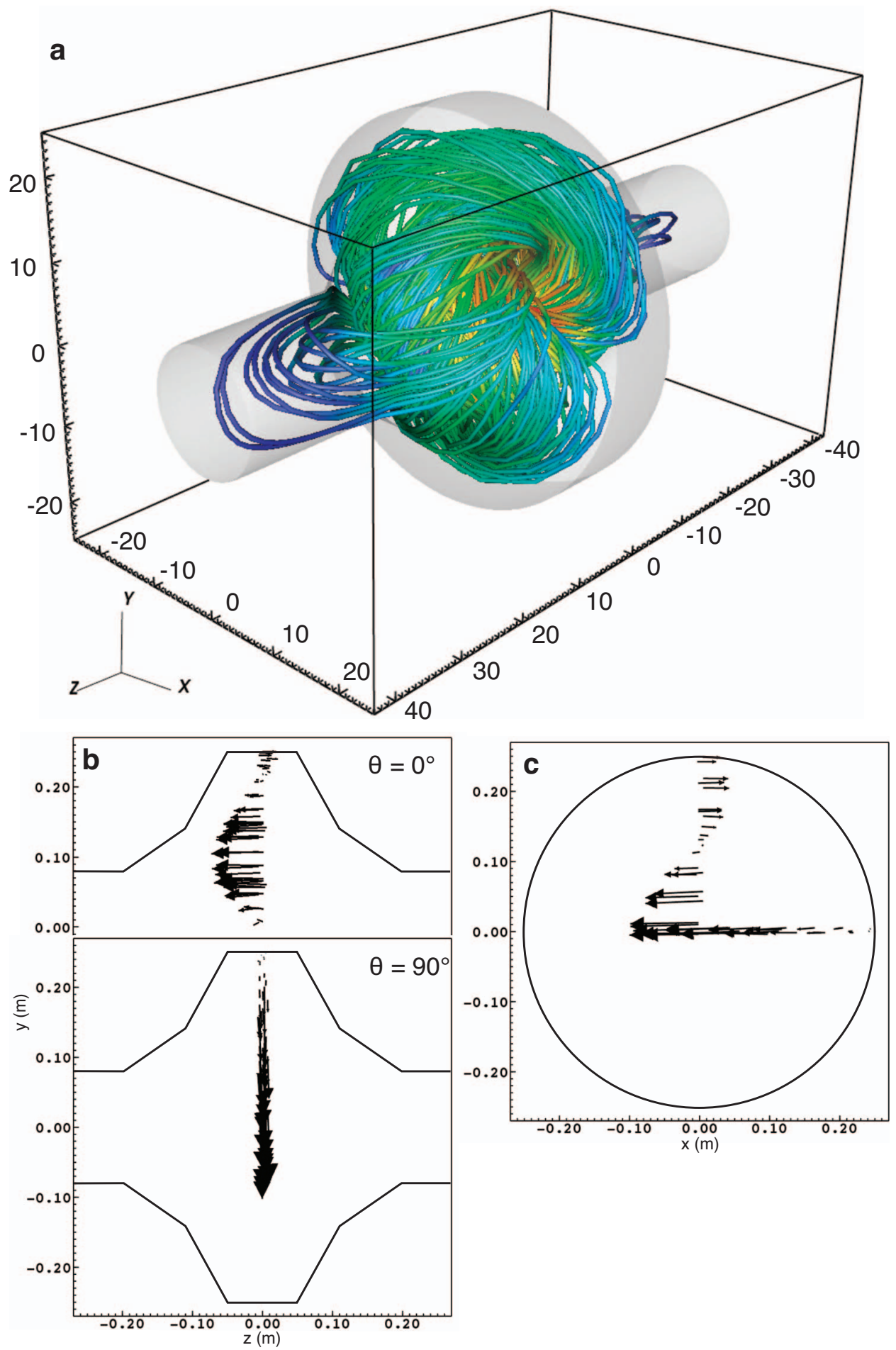

FIG. 9. (Color) The second eigenstate for Taylor relaxation in the oblate flux conserver in SSX, generated by the PSI-TET code. This is a tilted mode. In a, the flux conserver geometry is represented by the gray transparent solid, while the streamlines illustrate the magnetic fields, showing the overall structure of the state. Color represents $|\boldsymbol{B}|$. Magnetic field vectors are displayed in [(b) and (c)] with the poloidal field shown in (b) and the toroidal field in (c) compared with the measured field in Fig. 3.

not not be accurately calculated using this equation. The $\lambda$ profile for the observed state is flat and agrees with the calculated value, as shown in Fig. 7. The time evolution of $\lambda$ is shown in Fig. 8, where it can be seen that the plasma is in the minimum energy state during the period at 64-78 $\mu \mathrm{s}$. The $\lambda$ profile shown in Fig. 7 is taken from the end of this period.

The Taylor analysis shows that next eigenstate above the ground state is nonaxisymmetric, as is shown in Fig. 9. The second eigenmode with $\lambda=21.5 \mathrm{~m}^{-1}$ has only $0.6 \%$ more energy than the axisymmetric ground state, so they are nearly degenerate. Though the axisymmetric state is preferred, the tilted state is accessible in cohelicity merging due to the close spacing of the modes in energy. $\beta$ is low as calculated, but finite, so it might provide the impetus to select between 
the axisymmetric ground state and the tilted state. Magnetics obtained from experimental observations of the tilted mode are shown in Fig. 3 and compares favorably to second calculated eigenstate shown in Fig. 9. The $\lambda$ profile for nonaxisymmetric plasma is not flat and indicates that while the global structure of the plasma agrees roughly with the calculated field structure of the second lowest energy state, this state can be thought of as a metastable state. Even though the plasma lingers in this state for the duration of its measurable lifetime, the plasma is still evolving, albeit slowly. This state has a comparable lifetime to the axisymmetric state. The tilted states tend to align with one of the two quartz-clad magnetics probe stalks, suggestive of small nonideal effects introduced by the nonaxisymmetric probe stalks.

Continuous spheromak merging (even without direct reconnection as shown here) may be a useful way to sustain a large spheromak discharge. This pulsed sustainment has been observed in the Sustained Spheromak Plasma Experiment (SSPX), though in that case, the geometry of the merging is cohelicity but with direct reconnection of the poloidal fields. ${ }^{5}$ Even in the case without direct reconnection described here, long-lived merged states are achieved.

In conclusion, the observation and characterization has been performed of an axisymmetric spheromak formed by the dynamic merging of two smaller matched spheromaks of the same magnetic helicity in SSX. Despite being initially misaligned for magnetic reconnection, the two spheromaks merge with extensive nonaxisymmetric activity and emerge as a single quiescent spheromak containing most of the ini- tial helicity of the system, which then proceeds to decay slowly.

${ }^{1}$ T. R. Jarboe, Plasma Phys. Controlled Fusion 36, 945 (1994).

${ }^{2}$ C. G. R. Geddes, T. W. Kornack, and M. R. Brown, Phys. Plasmas 5, 1027 (1998).

${ }^{3}$ T. R. Jarboe, I. Henins, H. W. Hoida, R. K. Linford, J. Marshall, D. A. Platts, and A. R. Sherwood, Phys. Rev. Lett. 45, 1264 (1980).

${ }^{4}$ A. al-Karkhy, P. K. Browning, G. Cunningham, S. J. Gee, and M. G. Rusbridge, Phys. Rev. Lett. 70, 1814 (1993).

${ }^{5}$ S. Woodruff, B. W. Stallard, H. S. McLean, E. B. Hooper, R. Bulmer, B. I. Cohen, D. N. Hill, C. T. Holcomb, J. Moller, and R. D. Wood, Phys. Rev. Lett. 93, 205002 (2004).

${ }^{6}$ Y. Ono, M. Yamada, T. Akao, T. Tajima, and R. Matsumoto, Phys. Rev. Lett. 76, 3328 (1996)

${ }^{7}$ C. D. Cothran, A. Falk, A. Fefferman, M. Landreman, M. R. Brown, and M. J. Schaffer, Phys. Plasmas 10, 1748 (2003).

${ }^{8}$ M. R. Brown, Phys. Plasmas 6, 1717 (1999).

${ }^{9}$ J. B. Taylor, Phys. Rev. Lett. 33, 1139 (1974).

${ }^{10}$ M. Landreman, C. D. Cothran, M. R. Brown, M. Kostora, and J. T. Slough, Rev. Sci. Instrum. 74, 2361 (2003).

${ }^{11}$ C. D. Cothran, J. Fung, M. R. Brown, and M. J. Schaffer, Rev. Sci. Instrum. 77, 063504 (2006).

${ }^{12}$ V. H. Chaplin, M. R. Brown, D. H. Cohen, T. Gray, and C. D. Cothran, Phys. Plasmas 16, 042505 (2009).

${ }^{13}$ S. Chandrasekhar and L. Woltjer, Proc. Natl. Acad. Sci. U.S.A. 44, 285 (1958).

${ }^{14}$ M. Yamada, Y. Ono, A. Hayakawa, M. Katsurai, and F. W. Perkins, Phys. Rev. Lett. 65, 721 (1990).

${ }^{15}$ S. O. Knox, C. W. Barnes, G. J. Marklin, T. R. Jarboe, I. Henins, H. W. Hoida, and B. L. Wright, Phys. Rev. Lett. 56, 842 (1986).

${ }^{16}$ T. R. Jarboe, W. T. Hamp, G. J. Marklin, B. A. Nelson, R. G. O’Neill, A. J. Redd, P. E. Sieck, R. J. Smith, and J. S. Wrobel, Phys. Rev. Lett. 97, 115003 (2006). 